\title{
Review of: "Genetic analysis of early phenology in lentil identifies distinct loci controlling component traits"
}

\author{
Fanjiang Kong ${ }^{1}$ \\ 1 Guangzhou University
}

Potential competing interests: The author(s) declared that no potential competing interests exist.

In their manuscript, Vinodan Rajandran et al. excavate several QTLs related to flowering time and other traits in lentil, make an initial research on the genetic interaction between two major QTLs, and characterize their candidate genes in an ingenious way. These results benefit the research of genetic basis for the adaptation of lentils.

It's a very well-structured and well-written paper with a clear story, everything I could ask for is here.

1. Ordinates are needed in Fig.4B, meanwhile, number of plants in each lines/groups should be addressed in figure legend.

2. Further confirmation of the candidate genes is encouraged in following work.

3. The geographic distribution of the $7.4 \mathrm{~kb}$ deletion in the FTa1-FTa2 intergenic region implies its contribution to global adaptation in lentil. This part is an important content of the article. Thus, the world map in Supplementary Figure 10 is suggested to be move to main figures. 